\title{
AN EXPERIMENT ON THE NUMERICAL DAMPING OF MOVING PARTICLE SEMI-IMPLICIT (MPS) METHOD
}

\author{
F. K. Motezuki ${ }^{1}$, L.Y. Cheng ${ }^{2}$ \\ ${ }^{1}$ Numerical Offshore Tank, Escola Politécnica, University of São Paulo (fmote@tpn.usp.br) \\ ${ }^{2}$ Department of Construction Engineering, Escola Politécnica, University of São Paulo
}

\begin{abstract}
The Moving Particle Semi-Implicit (MPS) method is a lagrangian particle method originally developed to simulate incompressible flow. However, when applied to the study of some phenomena, a non negligible numerical damping of the particles motion was observed by the authors. In this work a simulator developed at the Numerical Offshore Tank at University of São Paulo based on the MPS method is used. To investigate the nature and the effects of the numerical damping, numerical experiments based on two benchmark cases with well known analytical solutions were carried out in the present work. The first case is the Poiseuille flow and the results show that for low acceleration values there is a numerical damping that, in extreme cases, can stop the flow; but for higher accelerations, agreement with the analytical profile of velocities were obtained. The second case is a falling-film flow, also the results show that the discrepancy between numerical and analytical results is remarkable in the cases with lower acceleration, but for higher accelerations there is a good agreement between both approaches.
\end{abstract}

Keywords: Moving Particle Semi-Implicit, Numerical method, Numerical damping.

\section{INTRODUCTION}

The Lagrangian particle methods are recognized for their ability to simulate non-linear hydrodynamics phenomena, such as those involve large free surface and boundary deformations, fragmentation and merging, complex geometries and multi-body problems that traditional Eulerian mesh based methods present shortcomings. As each particle carries with it physical quantities that can be evaluated with ease, the particle methods can also be applied to multi-physics problems.

The simulator used in this work was developed at the Numerical Offshore Tank at the University of São Paulo and is based on the Moving Particle Semi-Implicit (MPS) method [2], that is a lagrangian particle method originally developed to simulate incompressible flow. However, when applied to the study of some phenomena where the forces driving the phenomena are relatively small, such as in the natural convection, a non negligible numerical damping of the particles movement was observed. 
In order to investigate the nature and the effects of the numerical damping, the present work carried out a basic study by conducing numerical experiments using two benchmark cases with well known analytical solutions [4] for the transient behaviour of the flow. The results of both simulation cases were compared in an attempt to quantify the effect of the numerical damping as a numerical variation of the fluid viscosity.

In the first case the Poiseuille flow, that is the flow of a viscous fluid between two static horizontal plates, is analysed. Originally the fluid flow is stimulated by a pressure gradient between the ends of the channel; As the pressure boundary condition is not applicable in MPS for inflow and outflow boundaries, in the present study, the pressure gradient between the inlet and outlet is generated by an horizontal acceleration with the value equal to the horizontal component of gravity with the channel inclined by 2, 5, 7.5, 10 and 15 degrees.

The second case is a falling-film where a thin film of viscous fluid flows down over an inclined plate. Inclination angles of 2, 5, 7.5, 10 and 15 degrees were considered.

In the next section the MPS method is presented with the governing equations and details about the differential operators, boundary conditions and solution algorithm and then the cases of study and the results obtained.

\section{THE MPS METHOD}

\subsection{Governing Equations}

The governing equations of a incompressible flow are continuity equation, Eq. (1), and conservation of momentum, Eq. (2).

$$
\begin{gathered}
\nabla \cdot \vec{u}=0 \\
\frac{D \vec{u}}{D t}=-\frac{1}{\rho} \nabla P+\nu \nabla^{2} \vec{u}+\vec{F}
\end{gathered}
$$

where $\vec{u}$ is the velocity vector, $t$ is the time, $\rho$ is the fluid density, $P$ is the pressure, $\nu$ is the kinematic viscosity coefficient and $\vec{F}$ represents the external forces.

The Lagrangian approach of MPS avoids the numerical diffusion due to the advective terms. In the method the differential operators of the governing equations are replaced by numerical operators based on a particle interaction model that uses a kernel function given by

$$
w\left(\left|\vec{r}_{j}-\vec{r}_{i}\right|, r_{e}\right)=\left\{\begin{aligned}
\frac{r_{e}}{\left|\vec{r}_{j}-\vec{r}_{i}\right|}-1 & \text { if }\left|\vec{r}_{j}-\vec{r}_{i}\right|<r_{e} \\
0 & \text { if }\left|\vec{r}_{j}-\vec{r}_{i}\right| \geq r_{e}
\end{aligned}\right.
$$

where $\left|\vec{r}_{j}-\vec{r}_{i}\right|$ is the distance between the particle $i$ and its neighbors $j$ and $r_{e}$ is the effective radius and in this work is adopted $r_{e}=2.1 l_{0}$ where $l_{0}$ is the initial distance between particles. The sum of the weight function of a particle $i$ and its neighbors $j$ is called particle number density pnd and is defined as

$$
\text { pnd }=\sum_{j \neq i} w\left(\left|\vec{r}_{j}-\vec{r}_{i}\right|, r_{e}\right)
$$


The gradient of a scalar function $\phi$ is modeled in MPS as

$$
\langle\nabla \phi\rangle_{i}=\frac{d}{p n d^{0}} \sum_{j \neq i}\left[\frac{\phi_{j}-\phi_{i}}{\left|\vec{r}_{j}-\vec{r}_{i}\right|^{2}}\left(\vec{r}_{j}-\vec{r}_{i}\right) w\left(\left|\vec{r}_{j}-\vec{r}_{i}\right|, r_{e}\right)\right]
$$

where $d$ is the number of dimensions of the case being simulated and $p n d^{0}$ is the initial particle number density, calculated by Eq. (4) using the initial particle distribution.

The laplacian operator according to the original MPS method is given by

$$
\left\langle\nabla^{2} \phi\right\rangle_{i}=\frac{2 d}{p n d^{0} \lambda} \sum_{j \neq i}\left(\phi_{j}-\phi_{i}\right) w\left(\left|\vec{r}_{j}-\vec{r}_{i}\right|, r_{e}\right)
$$

where $\lambda$ is defined as

$$
\lambda=\frac{\sum_{j \neq i}\left|\vec{r}_{j}-\vec{r}_{i}\right|^{2} w\left(\left|\vec{r}_{j}-\vec{r}_{i}\right|, r_{e}\right)}{\sum_{j \neq i} w\left(\left|\vec{r}_{j}-\vec{r}_{i}\right|, r_{e}\right)}
$$

\subsection{Boundary Conditions}

\subsubsection{Wall boundary condition}

In MPS the walls are modeled using a layer of fixed particles followed by two layers of dummy particles that are used to complement the calculation of particle number density for the wall particles. This is necessary to obtain correct pressures and ensure the incompressibility of the model in the near wall region.

The Figure 1 shows the particle distribution near the wall, presenting the fluid, wall and dummy particles and the effective radius of the wall particle $i$.

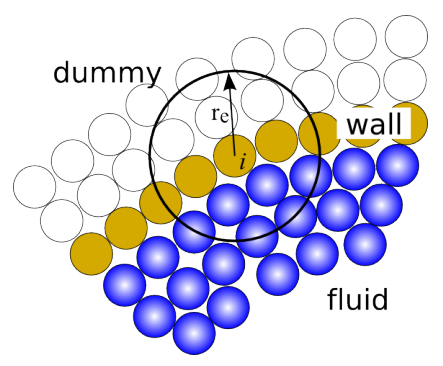

Figure 1. Detail from the near wall region and the effective radius $r_{e}$ from the particle $i$.

\subsubsection{Free surface}

The original condition [2] to detect free surface particles uses the calculated pnd compared with the initial particle number density with a full neighborhood $p n d^{0}$ and is given by

$$
\text { pnd }<\beta \cdot \text { pnd }^{0}
$$

where $\beta$ is a control value and is originally adopted as $\beta=0.97$. 
This is an efficient method to identify the free surface particles, but it is too sensitive to the little variations in the distance between the particle and its neighbors and is common to have particles in the middle of fluid identified as free surface.

Tanaka and Masunaga [5] attempted to overcome this issue by using a modified weight function given by

$$
w\left(\left|\vec{r}_{j}-\vec{r}_{i}\right|, r_{e}\right)= \begin{cases}1 & \text { if }\left|\vec{r}_{j}-\vec{r}_{i}\right|<r_{e} \\ 0 & \text { if }\left|\vec{r}_{j}-\vec{r}_{i}\right| \geq r_{e}\end{cases}
$$

this approach was significantly better but depending on the value of the control parameter $\beta$ two layers of particles are identified as free surface or many particles from the free surface are not identified as free surface in hydrodynamic cases. From experiments, an optimal value of $\beta=0.85$ was obtained.

This work uses a mixed approach [3] where the original free surface condition and the condition of Tanaka and Masunaga [5], both have to be satisfied to identify a particle as belonging to the free surface.

The use of both criteria enhances the process of identification in a way that only the particles actually at the free surface are identified as being free surface.

\subsubsection{Periodic boundary condition}

Periodic boundary conditions are applied in cases where a phenomenon repeats cyclically in space.

In the periodic boundary condition, illustrated as a dashed line in Fig. 2, when a particle flows out of the computational domain it is reintroduced with the same physical properties in the opposite side of the domain as illustrated in Fig. 2(a).

To calculate the differential operators of the particles near the periodic boundary condition, the particles in the opposite side are also considered. The Figure 2(b) shows the red particles that should be considered in the calculation of the differential operator of the green particle, but they are on the opposite side of the computational domain. The gray particles shows where the red particles should be for the calculation, so the red particles are numerically displaced by the size of the computational domain. This procedure make it easy to adapt the differential operators and also the linear equation system for the periodic boundary condition.

\subsection{Solution algorithm}

Inside each time-step the MPS separates the calculation in two stages, one explicit and other implicit.

In the explicit stage the velocity and position of the particles are calculated based on the viscous and external forces acting over the fluid.

The implicit stage solves the Poisson equation of pressure. To ensure the fluid incompressibility, the right hand side is calculated based on the difference between the particle number density estimated explicitly and the initial particle number density.

With the pressure, the corrections of velocity and position are carried out. 


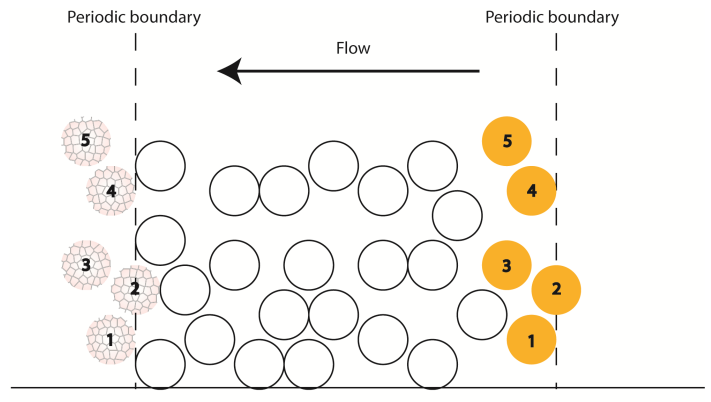

(a)

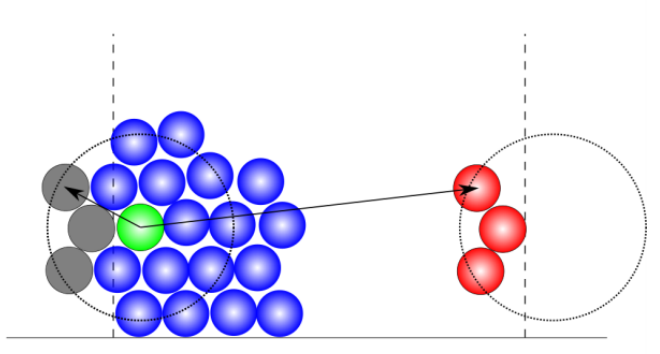

(b)

Figure 2. Details from the periodic boundary condition represented by the traced line. (a)The particles that flows out of the domain limited by the periodic boundary condition are repositioned in the opposite side. (b)The differential operators also consider the particles from the opposite side of the domain.

\section{CASES OF THE STUDY}

For the following simulations the considered fluid viscosity is $\nu=10^{-3}$ and the acceleration forces are equivalent to the inclination angles of 2, 5, 7.5, 10 and 15 degrees as shown in the table 1.

To perform the convergence analysis, simulations using four different particle distances, $0.0002,0.0005,0.0008$ and $0.001 \mathrm{~m}$ were carried out.

Table 1. Acceleration in $\mathrm{X}$ and $\mathrm{Y}$ direction for each inclination.

\begin{tabular}{ccc}
\hline Inclination angle & $\mathrm{X}$ acceleration & $\mathrm{Y}$ acceleration \\
\hline 2 & 0.342 & -9.804 \\
5 & 0.855 & -9.773 \\
7.5 & 1.280 & -9.726 \\
10 & 1.703 & -9.661 \\
15 & 2.539 & -9.476 \\
\hline
\end{tabular}

\section{RESULTS AND DISCUSSIONS}

\subsection{Poiseuille flow}

The Poiseuille flow simulated is a bidimensional flow between two infinite plates at $y=0$ and $y=L$ accelerated from the stationary condition by a uniform and constant force parallel to the $\mathrm{X}$ axis. The open ends of the simulation domain are periodic boundaries. Fig. 3 shows the schematic drawing of the simulated case.

The transient analytical solution is obtained from the integration of the momentum conservation equation $[1,4]$ and for this case is given by Eq. (10)

$$
v_{x}(y, t)=\frac{F}{2 \nu} y(y-L)+\sum_{n=0}^{\infty} \frac{4 F L^{2}}{\nu \pi^{3}(2 n+1)^{3}} \sin \left(\frac{\pi y}{L}(2 n+1)\right) \exp \left(\frac{-(2 n+1)^{2} \pi^{2} \nu}{L^{2}} t\right)
$$




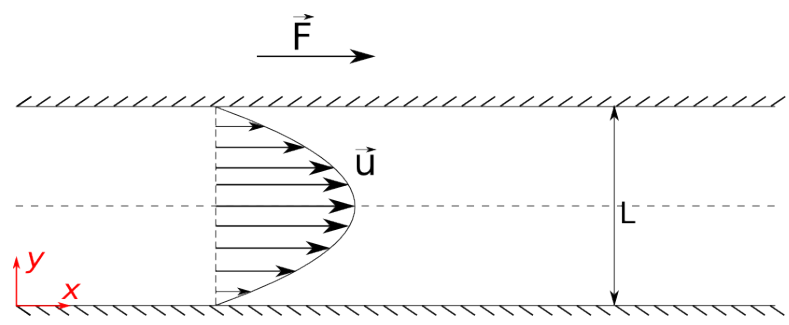

Figure 3. Schematic drawing of the Poiseuille flow case.

The velocity profiles obtained from the simulation and from the analytical equation are compared at the time $t=5 \mathrm{~s}$ when the steady flow condition is reached. The Figure 4 shows the 2 degrees and the 5 degrees case velocity profiles for the four particle distances and the analytical equation. Also the figure shows that for 2 degrees case the variation on the velocity profile is bigger than in the 5 degrees case.

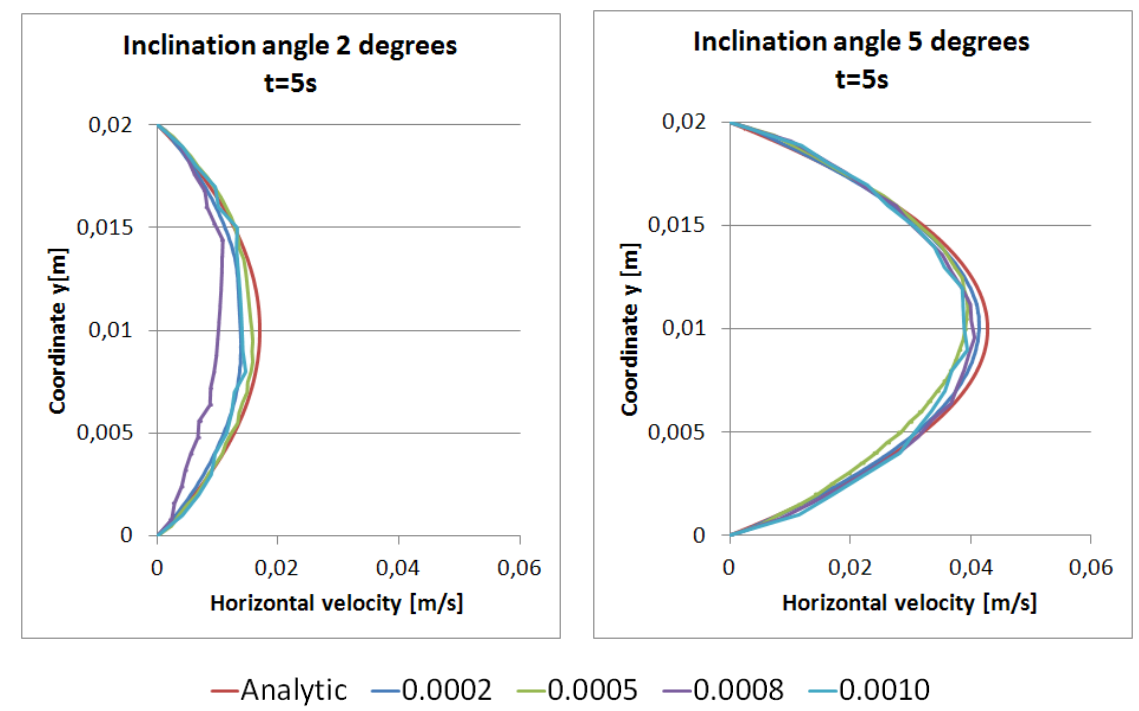

Figure 4. Velocity profile for different particle distances of the poiseuille flow case.

The Figure 5 shows that for the 2 degrees case, the error is very high and this error reduces as the angle increase. For the cases with inclination angle higher than 5 degrees, the error is limited around 5\%. At same time, the figure shows that in general, the error is higher for the cases with bigger particle distances.

Following the analysis of the simulations, it was observed that a dissipative behaviour is present and is more noticeable for low values of horizontal acceleration.

In an attempt to quantify this dissipation the simulation results were compared with the analytical equation added by a viscosity variation $\delta \nu=\nu_{\text {simulation }}-\nu_{\text {theoretical }}$. The viscosity variation is the one that minimizes the error between the simulation and the analytical results.

The absolute values of the viscosity variation are shown in the Fig. 6 and can be seen that for some cases the viscosity variation is positive or negative, i.e. a higher or lower viscous behaviour than that predicted theoretically, and the variation do not follow a pattern. Also from the Fig. 6 it is noticeable that the numerical approach presents shortcomings for 


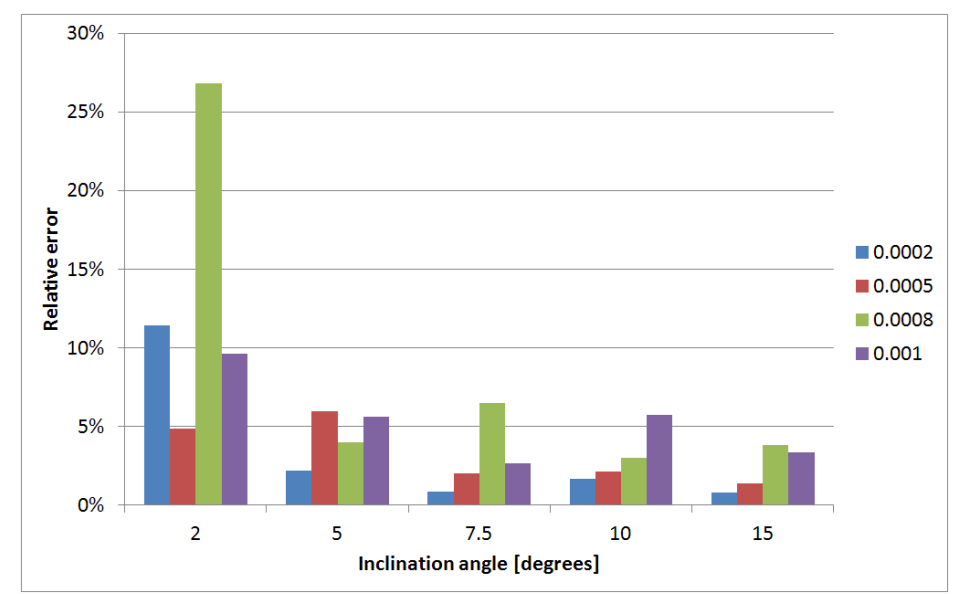

Figure 5. Mean squared error between the velocity profiles obtained from simulation and the analytical solution.

the 2 degree case and for the other cases the particle distance of $0.0008 \mathrm{~m}$ is the most critical resolution for the simulation.

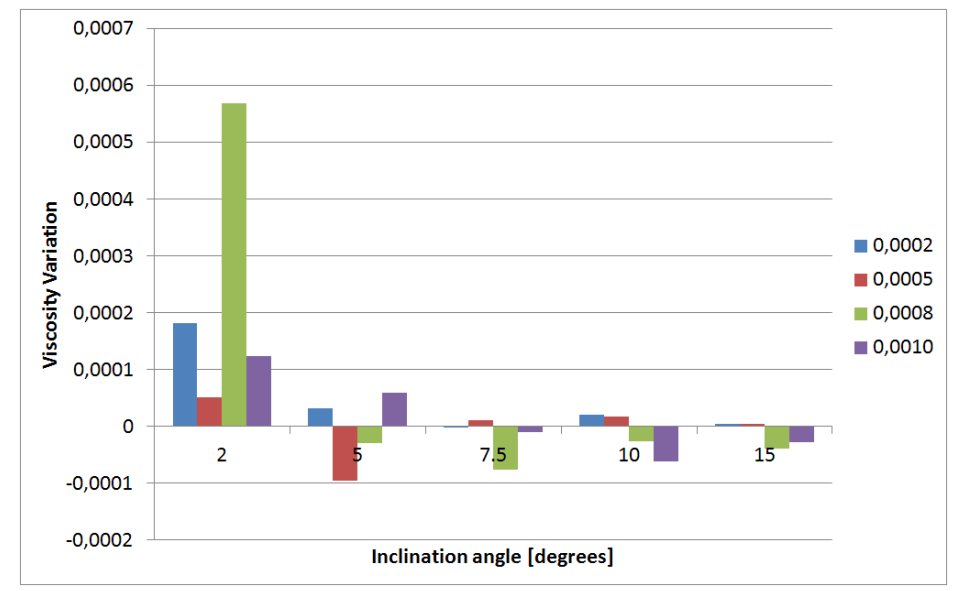

Figure 6. Viscosity variation of the poiseuille flow case.

\subsection{Falling film flow}

The falling film simulation consists of an inclined plate with a thin film of fluid that flows due to the gravitational force. In this case the periodic boundary condition is applied in the left and right border of the computational domain. Also the same five angles and four particle distances used in the previous case were simulated.

The Figure 7(a) shows an example of falling film device and the control volume used for the analysis of the case. The control volume is aligned to the inclined surface considering that the hydrostatic pressure in the film is negligible. In this way, only the tangential component of the gravity acceleration have influence in the phenomena, this configuration makes easy the deduction of the analytical equation.

The Figure 7(b) show a schematic drawing of the case used in the deduction of the analytical equation. 


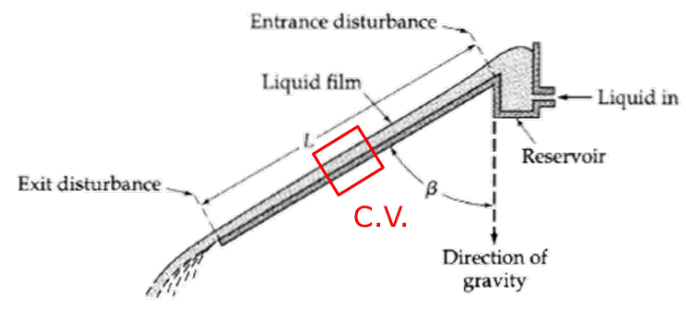

(a)

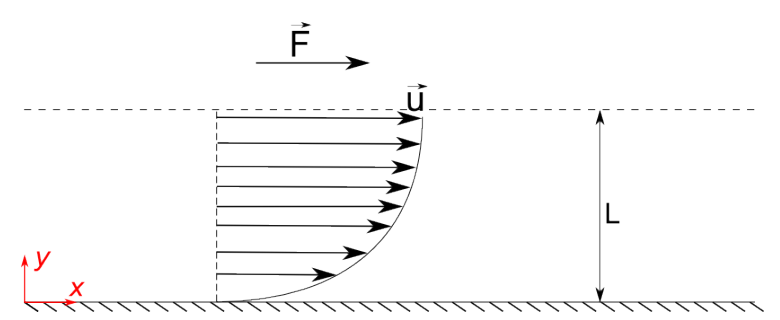

(b)

Figure 7. (a) A example of a falling film device. The C.V. is the control volume used for the analysis of this case. (b) Schematic used for the calculation of the analytical equation.

Other hypothesis used in the derivation of the analytical equation is that the flow is laminar and the surface remains undisturbed [1]. The analytical solution for the transient behaviour of this case is given by Eq. (11)

$$
v_{x}(y, t)=\frac{F}{2 \nu} y(y-2 L)+\sum_{n=0}^{\infty} \frac{16 F L^{2}}{\nu \pi^{3}(2 n+1)^{3}} \sin \left(\frac{\pi y}{2 L}(2 n+1)\right) \exp \left(\frac{-(2 n+1)^{2} \pi^{2} \nu}{4 L^{2}} t\right)
$$

It is worth to mention that for this case there is a transition of fluid regime with higher velocities. When the transition occurs, the above equation is no more valid to describe this flow, but for the initial instants it provides a good approximation.

Figure 8 shows the horizontal velocity profiles for the 2 degrees and 5 degrees case. It is remarkable that the 2 degrees have a higher variation of the velocity profile than with 5 degrees. Also for the 2 degrees case the figure shows that the shape of the velocity profile as particle distance increase is very similar to a increase in the viscosity of the fluid.

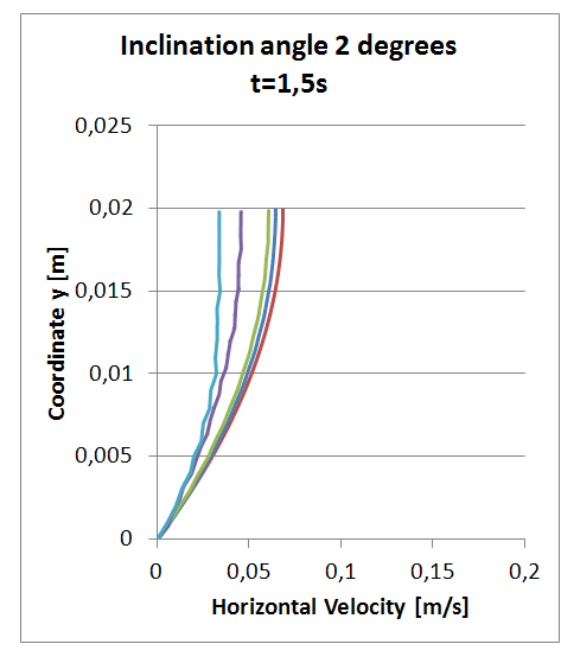

(a)

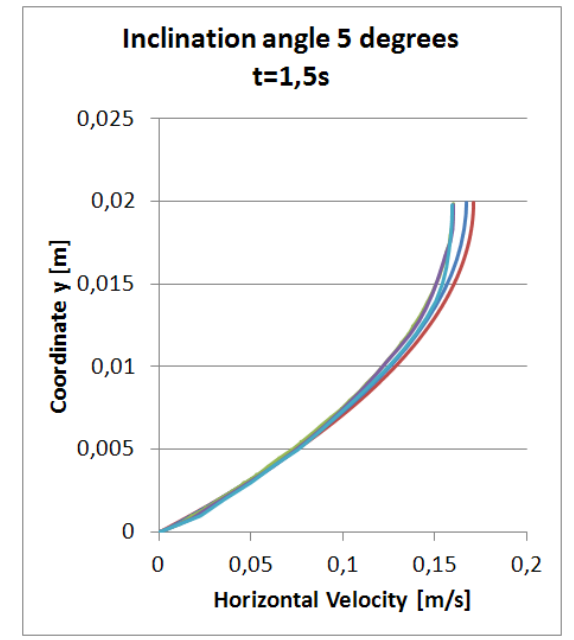

(b)

-Analytic $-0.0002-0.0005-0.0008-0.0010$

Figure 8. Velocity profile for the different particle distances of the poiseuille flow case. 
The Figure 9 shows the mean squared error between the simulation and the analytical solution taken at time $t=1.5 \mathrm{~s}$. For the 2 degrees case the error is relatively large and it is remarkable the large increase of the error when the particle distance is increased. This direct relation between error and particle distance is not obtained in other inclination angles. Moreover the error for inclination angle larger or equal to 5 degrees are lower than $5 \%$.

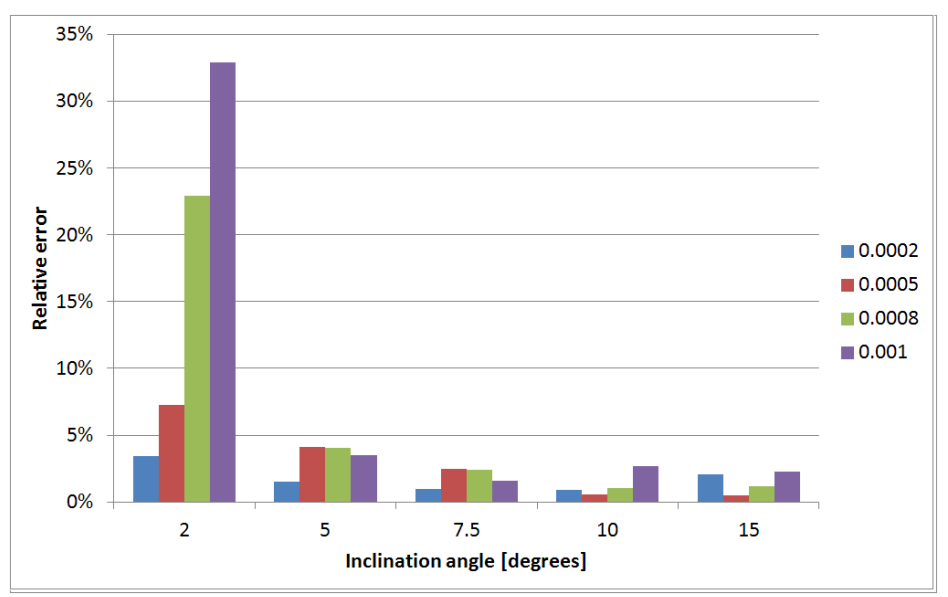

Figure 9. The mean squared error for the falling film case.

Also in this case the variation in the viscosity was calculated and the results in the Fig. 10 show that there is no pattern for the positive-negative variation and in general the bigger particle distances have a bigger viscosity variation.

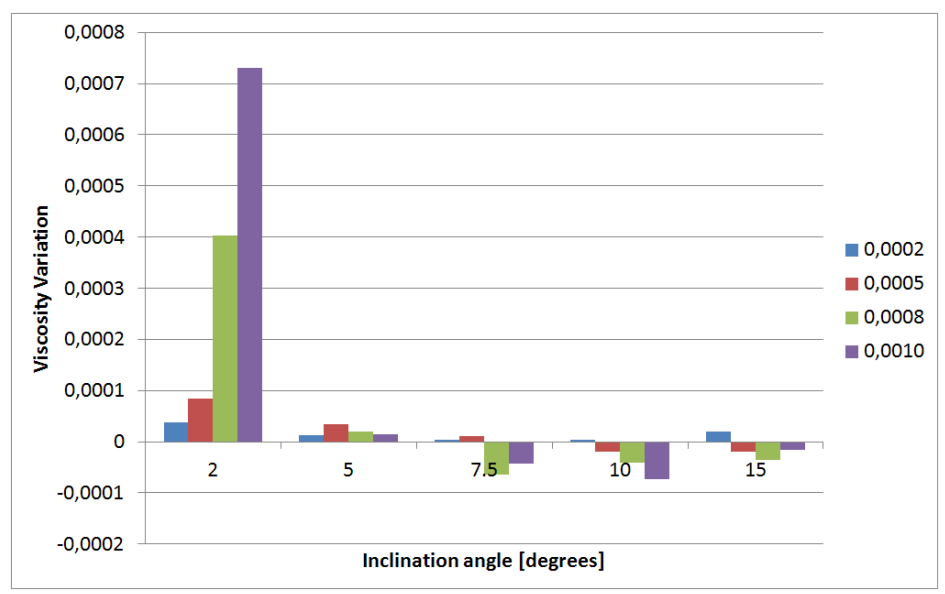

Figure 10. Viscosity variation of the falling-film case.

\section{Final remarks}

A dissipative behaviour was observed in this work for the lower acceleration forces, which indicates the existence of a relationship between the forces driving the phenomena and the dissipation.

Also in this work was shown that the positive-negative variation of the viscosity does not follow a pattern, but the variation amplitude decreases with the increase of acceleration forces. 
The results show that the numerical damping may not be negligible when simulating phenomena where the driving forces are low. As this numerical damping increases when the component of acceleration in the direction perpendicular to the flow increases, and do not occurs when gravity is neglected in the calculation, it seems to be more critical in gravity driven problems. A deeper study should be carried to determine the origins and quantify the numerical damping.

\section{Acknowledgements}

The author would like to express their gratitude to PETROBRAS for financial support on the development of the MPS-based simulation code at the Numerical Offshore Tank (TPN-USP).

\section{REFERENCES}

[1] Bird R.B., Stewart W.E., Lightfoot E.N., "Transport Phenomena", 2nd Ed., 2001.

[2] Koshizuka S., Oka Y., "Moving particle semi-implicit method for fragmentation of incompressible fluid". Nuclear science and engineering, 123(3), 421-434, 1996.

[3] Lee B.-H., Park J.-C., Kim M.-H., "Two-Dimensional Vessel-Motion/Liquid-Sloshing Interactions and Impact Loads by Using a Particle Method". Proceedings of the ASME 29th International Conference on Ocean, Offshore and Arctic Engineering (OMAE2010), Shanghai, 2010.

[4] Morris J.P., Fox P.J., Zhu Y., "Modeling Low Reynolds Number Incompressible Flows Using SPH”. Journal of Computational Physics, 136(1), 214-226, 1997.

[5] Tanaka M., Masunaga T., "Stabilization and smoothing of pressure in MPS method by Quasi-Compressibility”. Journal of Computational Physics, 229(11), 4279-4290, 2010. 